Physics

Physics Research Publications

Comment on "Anomalies in electrostatic calibrations for the measurement of the

Casimir force in a sphere-plane geometry"
R. S. Decca*
E. Fischbach ${ }^{\dagger}$
G. L. Klimchitskaya $a^{\ddagger}$
D. E. Krause**
D. Lopez ${ }^{\dagger \dagger}$
U. Mohideen ${ }^{\ddagger \ddagger}$
V. M. Mostepanenko ${ }^{\S}$

$*$
$\dagger$
$\ddagger$
$* *$
$\dagger \dagger$
$\ddagger \ddagger$
$\S$

This paper is posted at Purdue e-Pubs.

http://docs.lib.purdue.edu/physics_articles/1004 


\title{
Comment on "Anomalies in electrostatic calibrations for the measurement of the Casimir force in a sphere-plane geometry"
}

\author{
R. S. Decca, ${ }_{1}^{1}$ E. Fischbach, ${ }^{2}$ G. L. Klimchitskaya, ${ }^{3, *}$ D. E. Krause, ${ }^{4,2}$ D. López, ${ }^{5}$ U. Mohideen, ${ }^{6}$ and V. M. Mostepanenko ${ }^{3, \dagger}$ \\ ${ }^{1}$ Department of Physics, Indiana University-Purdue University Indianapolis, Indianapolis, Indiana 46202, USA \\ ${ }^{2}$ Department of Physics, Purdue University, West Lafayette, Indiana 47907, USA \\ ${ }^{3}$ Center of Theoretical Studies and Institute for Theoretical Physics, Leipzig University, D-04009 Leipzig, Germany \\ ${ }^{4}$ Department of Physics, Wabash College, Crawfordsville, Indiana 47933, USA \\ ${ }^{5}$ Center for Nanoscale Materials, Argonne National Laboratory, Argonne, Illinois 60439, USA \\ ${ }^{6}$ Department of Physics and Astronomy, University of California-Riverside, Riverside, California 92521, USA
}

(Received 21 September 2008; published 27 February 2009)

\begin{abstract}
Recently Kim et al. [Phys. Rev. A 78, 020101(R) (2008)] performed electrostatic calibrations for a plane plate above a centimeter-size spherical lens at separations down to 20-30 nm and observed "anomalous behavior." It was found that the gradient of the electrostatic force does not depend on separation as predicted on the basis of a pure Coulomb contribution. Some hypotheses which could potentially explain the deviation from the expected behavior were considered, and qualitative arguments in favor of the influence of patch surface potentials were presented. We demonstrate that for the large lenses at separations of a few tens of nanometers from the plate, the electrostatic force law used by the authors is not applicable due to possible deviations of the mechanically polished and ground lens surface from a perfect spherical shape. A model is proposed which provides a possible explanation for the observed anomalous behavior using the standard Coulomb force.
\end{abstract}

DOI: 10.1103/PhysRevA.79.026101

PACS number(s): 12.20.Fv, 03.70.+k, 04.80.Cc, 11.10.Wx

In Ref. [1], anomalies in the electrostatic calibration for the measurement of the Casimir force in a sphere-plane geometry were found. Precision electrostatic calibrations in the sphere-plane geometry have attracted much attention in the last few years in connection with measurements of the Casimir force [2-16]. In these measurements electrostatic calibrations play an important role. They allow precise independent determination of such basic quantities as absolute separation, cantilever spring constants, sphere radii, parameters of the micromechanical oscillator, and the contact potential difference of the grounded test bodies. Because of this, any inaccuracy in the theoretical expression for the electric force used in the calibration introduces additional systematic errors in the measurement data for the Casimir force and invites questions on the validity of the experimental results that are obtained.

Reference [1] presents the experimental data from electrostatic calibrations in the configuration of a Si plate above a large spherical lens of radius $R=30.9 \pm 0.15 \mathrm{~mm}$, both covered with an Au film. In these calibrations, separation distances $d$ down to a few tens of nanometers from the point of contact between the plate and the sphere were explored. Surprisingly, instead of the expected $d^{-2}$ distance dependence of the gradient of the electric force, as is given by the main contribution to the exact result in the sphere-plate configuration [11] or, equivalently, by the proximity force approximation, a dependence of the order $d^{-1.7}$ was observed from four separate experimental sequences. The values of the contact

\footnotetext{
*On leave from North-West Technical University, St. Petersburg, Russia.

†On leave from Noncommercial Partnership "Scientific Instruments," Moscow, Russia.
}

potential difference $V_{c}$, in at least two sequences, were found to be separation dependent. Reference [1] discusses five hypotheses which could potentially explain a deviation from the expected force law, specifically, static deflection of the cantilever, thermal drift, nonlinearity of the piezoelectric transducer, nonlinear oscillations of the cantilever, and the surface roughness. It was found that none of these explain the anomaly. A sixth hypothesis, favored by the authors, is the effect of patch surface potentials. However, no specific arguments in its favor were provided, except for the observation that $V_{c}$ is separation dependent in at least two sequences. This is, however, simply an observation that the electric force gradient behaves anomalously, rather than a determination of the specific physical cause. On this basis the authors argue that their "findings affect the accuracy of the electrostatic calibrations and invite reanalysis of previous determinations of the Casimir force."

Below we demonstrate that the observed anomalies can be explained using the standard distance dependence of the electric force, if one takes into account deviations of the lens surface from a perfect spherical shape. Such deviations are unavoidably present on any spherical surface of centimeter size. Hence, they preclude the use of the simplest formulation of the proximity force approximation for a constant radius of curvature at short separations as used in the paper. In the conclusion we formulate some basic requirements for precision calibration procedures and emphasize that all previous experiments on the measurement of the Casimir force [2-16] are irrelevant to the phenomenon observed in [1] because they are performed at large separations [2], or with spheres of much smaller radii [3-16].

Using the proximity force approximation [17], Ref. [1] represents the gradient of the electric force between a centimeter-size spherical lens and plate as 


$$
F_{\mathrm{el}}^{\prime}=\pi \epsilon_{0} \frac{R\left(V-V_{c}\right)^{2}}{d^{2}},
$$

where $V$ is the applied voltage, $V_{c}$ is the contact potential, $d$ is the gap separation, and $\epsilon_{0}$ is the permittivity of vacuum. (The minus sign on the right-hand side of this formula in [1] is a misprint.) The frequency shift of the cantilever due to an external force is given by

$$
\nu^{2}-\nu_{0}^{2}=-\frac{1}{4 \pi^{2} m_{\mathrm{eff}}} F_{\mathrm{el}}^{\prime},
$$

where $m_{\text {eff }}$ is the effective mass of the oscillator. Using Eq. (1), this frequency shift can be rearranged to the form

$$
\nu^{2}-\nu_{0}^{2}=-k_{\mathrm{el}}(d)\left(V-V_{0}\right)^{2}, \quad k_{\mathrm{el}}(d)=\frac{\epsilon_{0} R}{4 \pi m_{\mathrm{eff}} d^{2}} .
$$

However, as noted in [1], the experimental data from four separate sequences follow a power law, similar to the $d^{-2}$ dependence in Eqs. (1) and (3), but with powers $-1.70 \pm 0.01,-1.77 \pm 0.02,-1.80 \pm 0.01$, and $-1.54 \pm 0.02$, which are far from the expected value of -2 .

As mentioned above, Ref. [1] discusses several hypotheses which could explain the observed anomaly and discards all of them. As a possible explanation the effect of patch surface potentials was considered, but only qualitative arguments that this effect might be responsible for the observed anomalous behavior of the electrostatic force were provided. These arguments, however, do not take into account Refs. $[8,10]$, where the role of patches due to the grains of polycrystalline metal film in the measurements of the Casimir force by means of an atomic force microscope [8] and a micromechanical torsional oscillator [10] was specifically investigated in detail. Thus, in [8] it was concluded that the electric force due to patch potentials of this type contributes only $0.23 \%$ and $0.008 \%$ of the Casimir force at separations $d=62 \mathrm{~nm}$ (the closest separation in this experiment) and 100 $\mathrm{nm}$, respectively. These results are based on the theoretical expressions of Ref. [18] and on the determination of the maximum and minimum sizes of grains in gold layers covering the test bodies using the atomic force microscopy images of the surfaces of the plate and sphere. With respect to the electric force $F_{\text {el }}$ due to the applied potential $V=0.2 \mathrm{~V}$, the patch effect contributes only $0.064 \%$ and $0.0011 \%$ at separations of 62 and $100 \mathrm{~nm}$, respectively (in this experiment the contact potential was determined to be $V_{c}$ $=3 \pm 3 \mathrm{mV}$ ). According to the analysis of Ref. [10], at the shortest separations, $d=160$ and $170 \mathrm{~nm}$ in the experiment using a micromechanical oscillator, patch potentials contribute only $0.037 \%$ and $0.027 \%$ of the Casimir pressure. With respect to the electric pressure $P_{\text {el }}$ due to $V-V_{c}=0.2 \mathrm{~V}$, here the patch effect contributes $0.19 \%$ and $0.13 \%$ at $d=160$ and $170 \mathrm{~nm}$, respectively. There is another type of patch potential due to scratches, adsorbates, chemical contaminants, and dust on the surface which depends on the applied voltage and, thus, significantly influences the calibration measurements making $V_{c}$ separation dependent. It is generally recognized that such poor-quality samples should not be used in precision experiments on the Casimir force. Thus, it is un-

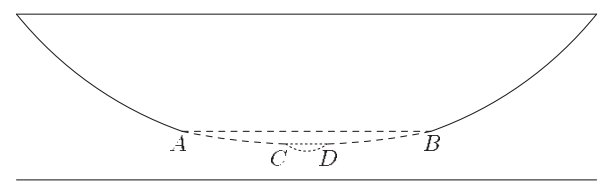

FIG. 1. Model of the surface of the spherical lens of radius $R$ with local deviations from perfect shape (see text for detail). Figure is not to scale.

likely that patch charges are responsible for the anomalous distance dependence of the gradient of the electric force observed in Ref. [1].

Here, we present an alternative explanation for the observation of Ref. [1] that the power of the distance in the gradient of the electric force differs from -2. A key point to note is that Ref. [1] used very large spheres of radius more than 3 $\mathrm{cm}$, which approached as close as $20-30 \mathrm{~nm}$ to the plate. In such a situation the proximity force approximation in the form (1) is not valid. To see this we note that Eq. (1) was derived for a perfect spherical lens with a constant curvature radius $R$ at each point of the surface. Reference [1] mentions the deviations from ideal spherical geometry and its possible role at the smallest distances but considers this only in connection with the surface roughness. Using the measured rms values of roughness from 1 to $2 \mathrm{~nm}$, the authors find the respective corrections negligible. In reality, however, surfaces of large lenses are far from perfect, even excluding the rms roughness from consideration. In particular, the typical surface quality of centimeter-size surfaces is usually characterized in terms of the scratch and dig optical surface specification data. This means that depending on the quality of lens used, bubbles or pits with a maximal diameter varying from $30 \mu \mathrm{m}$ to $1.2 \mathrm{~mm}$ are allowed on the surface. There may also be scratches on the surface with a width varying from 3 to $120 \mu \mathrm{m}$ [19]. Surface accuracy is characterized by the power and irregularity, where power defines the deviation of the fabricated surface radius from the radius of a test surface. When the separation distance between the sphere and the plate is sufficiently large, the deviations from perfect spherical shape can be neglected. Only in this case is the global curvature radius $R$ important. At short separations, however, local radii of curvature, which may differ from the global radius by several orders of magnitude due to the mechanical polishing and grinding of glass lens, contribute significantly to the result.

Based on the above information, we present in Fig. 1 a model of a spherical lens of radius $R$ containing a region $\mathrm{AB}$ of a larger curvature radius $R_{\mathrm{AB}}=1.6 R=49.4 \mathrm{~mm}$ and a spherical bubble of $R_{\mathrm{CD}}=30 \mu \mathrm{m}$ radius. We emphasize that the height of sector $\mathrm{AB}$ is $H=250 \mathrm{~nm}$ and the height of sector CD is $h=8 \mathrm{~nm}$. The imperfections in the large spherical surface, as shown (not to scale) in Fig. 1, are well below the error in the determination of the lens radius $\Delta R$ $=0.15 \mathrm{~mm}$. Thus, for a perfect sphere of radius $R$, sector $\mathrm{AB}$ would have height $\widetilde{H}=400 \mathrm{~nm}$. This means that the maximum flattening of the spherical surface in region $\mathrm{AB}$ is only $150 \mathrm{~nm}$, i.e., $0.1 \%$ of the allowed error $\Delta R$ in the radius $R$.

The application of the proximity force approximation to the configuration in Fig. 1 at small separations results in the modified coefficient 

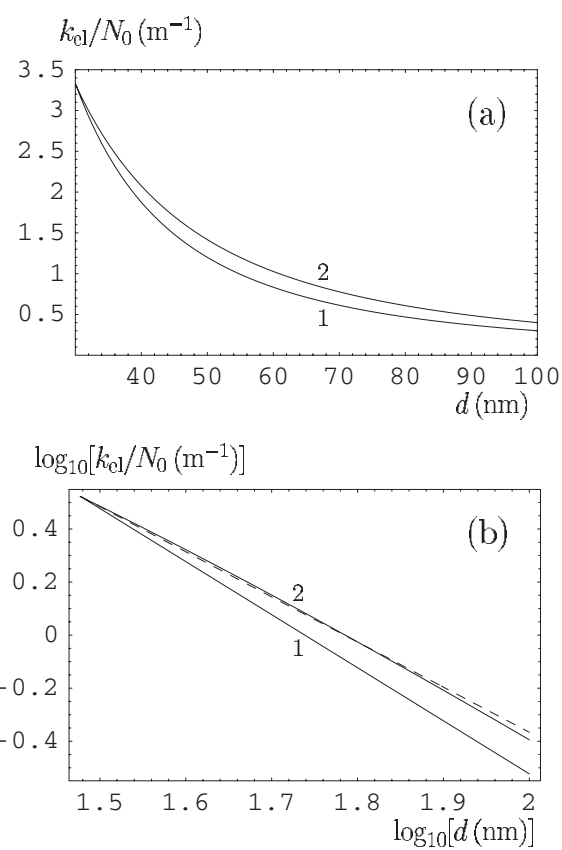

FIG. 2. The normalized coefficient $k_{\mathrm{el}}$ in (a) natural and (b) double logarithmic scales as function of separation. Solid lines 1 and 2 indicate $k_{\mathrm{el}}$ and $k_{\mathrm{el}}^{\mathrm{mod}}$ for a perfect sphere and for a sphere with local deviations from perfect sphericity. The dashed line demonstrates $\tilde{k}_{\mathrm{el}}$ decreasing as $d^{-1.7}$.

$$
k_{\mathrm{el}}^{\mathrm{mod}}(d)=\frac{\epsilon_{0}}{4 \pi m_{\mathrm{eff}}}\left[\frac{R_{\mathrm{CD}}}{d^{2}}+\frac{R_{\mathrm{AB}}-R_{\mathrm{CD}}}{(d+h)^{2}}-\frac{R_{\mathrm{AB}}-R}{(d+h+H)^{2}}\right] .
$$

Numerically, $k_{\mathrm{el}}^{\mathrm{mod}}\left(d_{0}\right)=k_{\mathrm{el}}\left(d_{0}\right)$ at $d_{0}=30 \mathrm{~nm}$. This equation means that the gradient of the electric force depends on the separation distance in a far different way than in Eq. (1). As an illustration, in Fig. 2(a) we plot the normalized coefficients $k_{\mathrm{el}}$, as given by Eq. (3) (solid line 1), and $k_{\mathrm{el}}^{\mathrm{mod}}$, as given by Eq. (4) (solid line 2), as functions of separation. The normalization factor is equal to $N_{0} \equiv \epsilon_{0} /\left(4 \pi m_{\text {eff }}\right)$ $\times 10^{13}$. It can be seen that there is a significant deviation between the coefficients obtained for a perfect spherical lens and that for the surface shown in Fig. 1. To describe this deviation quantitatively, in Fig. 2(b) we plot the same lines 1 and 2 in a double-logarithmic scale. In the same figure the dashed line shows the dependence of $\tilde{k}_{\mathrm{el}} / N_{0}$ on separation in accordance with

$$
\tilde{k}_{\mathrm{el}}(d)=\frac{\epsilon_{0} R}{4 \pi m_{\mathrm{eff}} d_{0}^{0.3} d^{1.7}}
$$

This expression having a power of -1.7 instead of -2 is shown in Ref. [1] to be consistent with the experimental data of the measurements of the electric force between a large lens and a plate at small separation distances. As is seen in Fig. 2(b), the experimentally consistent dependence (5) is well reproduced by the solid line 2 obtained using the standard electric force gradient taking into account local deviations from a perfect spherical shape, as presented in Fig. 1.

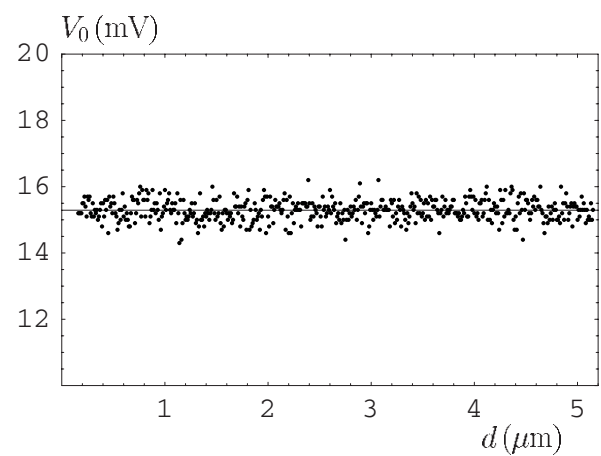

FIG. 3. Calibration results for the contact potential $V_{c}$ at different separations are shown as dots. The solid line indicates the mean value $V_{c}=15.29 \mathrm{mV}$ with the standard deviation equal to $0.13 \mathrm{mV}$.

We emphasize that Fig. 1 shows only one crude model of possible deviations from sphericity specific for large spherical surfaces. In precision measurements one should carefully investigate the interaction region of the large spherical surface microscopically and compute the electric force numerically by solving Poisson's equation (as done in Ref. [16]). These complications do not arise when using spheres with much smaller radii. Specifically, the surfaces of polystyrene spheres of about $100 \mu \mathrm{m}$ radius made from liquid phase are extremely smooth due to surface tension. The investigation of the surface quality of such spheres using a scanning electron microscope did not reveal any scratches or bubbles. However, the same investigation has shown the presence of bubbles in some $300 \mu \mathrm{m}$ and larger polystyrene spheres.

In precision electrostatic calibrations, as a part of experiments on measuring the Casimir force, the following rule is helpful. Depending on the size and quality of a spherical body, the minimal separation distance should be chosen in such a way that the contact potential $V_{c}$ and other basic quantities determined from calibration do not depend on separation where the calibration procedure is performed. As an example, in Fig. 3 we present previously unpublished calibration data for $V_{c}$ in the experiment on the indirect dynamic determination of the Casimir pressure between two parallel plates by means of a sphere oscillating above a micromechanical torsional oscillator [14]. In this experiment, a sapphire sphere of $R=151.3 \pm 0.2 \mu \mathrm{m}$ radius was used and the measurements of the Casimir pressure were performed over the separation range from 162 to $746 \mathrm{~nm}$. In Fig. 3 the calibration results for $V_{c}$ obtained at 500 different separation distances ranging from 160.4 to $5150.1 \mathrm{~nm}$ are shown as dots as a function of separation. It is seen that the results do not depend on separation over a wide separation region including the entire measurement range of the Casimir pressure. This confirms that proportions between the sphere radius and the minimum separation are determined correctly. The resulting mean contact potential is $V_{c}=15.29 \mathrm{mV}$ with the standard deviation equal to $0.13 \mathrm{mV}$. The equality $V_{c}=$ const can also be considered as an indirect confirmation of the fact that the interacting regions of the surfaces are clean.

One more important requirement for precision measurements of the Casimir force is that the piezo creep and drift should be calibrated and subtracted. In contrast to experiment [5], where continuous voltages were applied to the pi- 
ezo crystal which was interferometrically calibrated, or to experiments $[10,14]$, where the piezo crystal was monitored interferometrically with a feedback, Ref. [1] applies to the piezo crystal only static voltages and takes $8-10$ min to make a measurement. Then the creep is measured at some large voltage and is scaled linearly for the measurement time. This procedure may lead to errors because the piezo drift is nonlinear with the applied voltage, which might be critical at short separation distances.

One can conclude that contrary to the conclusion of Ref. [1] the observed "anomalies" are irrelevant to the precision experiments on measuring the Casimir force $[2,3,6,7]$ mentioned in [1] and all other performed experiments previously using the sphere-plate configuration $[4,5,8-16]$. The experimental precision of these experiments, and the measure of agreement of the obtained results with theory, remain the same as was stated in the original publications after taking account of subsequently published corrections, improvements, and reanalyses using more rigorous statistical approaches. (For example, the experimental data of Ref. [5] were later reanalyzed in Ref. [20].) It should be mentioned that Ref. [1] incorrectly ascribes the claimed accuracy from $0.1 \%$ to $5 \%$ to the experiments $[2,3,6,7]$. In fact the claimed accuracy of these experiments ranges from $1 \%$ to 5\%. Presently the most precise determination of the Casimir pressure using a micromechanical oscillator is characterized by an experimental error of $0.2 \%$ and by a $1.9 \%$ measure of agreement between experiment and theory at the shortest separation of $162 \mathrm{~nm}$ [14]. This experiment, however, is not mentioned in Ref. [1].

The above remarks demonstrate that the anomalous behavior of the electrostatic signal observed in Ref. [1] has a clear explanation in the mistaken assumption of a perfect spherical shape for a mechanically polished and ground large glass lens at nanoscale distances from a plate.

R.S.D. acknowledges NSF support through Grants No. CCF-0508239 and No. PHY-0701636 and from the Nanoscale Imaging Center at IUPUI. E.F. was supported in part by DOE under Grant No. DE-76ER071428. U.M., G.L.K., and V.M.M. were supported by the NSF Grant No. PHY0653657 (computations of the electric force) and DOE Grant No. DEFG02-04ER46131 (precise procedures for piezo calibrations). G.L.K. and V.M.M. were also partially supported by Deutsche Forschungsgemeinschaft under Grant No. 436 RUS 113/789/0-4.
[1] W. J. Kim, M. Brown-Hayes, D. A. R. Dalvit, J. H. Brownell, and R. Onofrio, Phys. Rev. A 78, 020101(R) (2008).

[2] S. K. Lamoreaux, Phys. Rev. Lett. 78, 5 (1997); 81, 5475(E) (1998).

[3] U. Mohideen and A. Roy, Phys. Rev. Lett. 81, 4549 (1998); G. L. Klimchitskaya, A. Roy, U. Mohideen, and V. M. Mostepanenko, Phys. Rev. A 60, 3487 (1999).

[4] A. Roy, C.-Y. Lin, and U. Mohideen, Phys. Rev. D 60, 111101(R) (1999).

[5] B. W. Harris, F. Chen, and U. Mohideen, Phys. Rev. A 62, 052109 (2000).

[6] H. B. Chan, V. A. Aksyuk, R. N. Kleiman, D. J. Bishop, and F. Capasso, Science 291, 1941 (2001); Phys. Rev. Lett. 87, 211801 (2001).

[7] R. S. Decca, D. López, E. Fischbach, and D. E. Krause, Phys. Rev. Lett. 91, 050402 (2003); R. S. Decca, E. Fischbach, G. L. Klimchitskaya, D. E. Krause, D. López, and V. M. Mostepanenko, Phys. Rev. D 68, 116003 (2003).

[8] F. Chen, G. L. Klimchitskaya, U. Mohideen, and V. M. Mostepanenko, Phys. Rev. A 69, 022117 (2004).

[9] M. Lisanti, D. Iannuzzi, and F. Capasso, Proc. Natl. Acad. Sci. U.S.A. 102, 11989 (2005).

[10] R. S. Decca, D. López, E. Fischbach, G. L. Klimchitskaya, D. E. Krause, and V. M. Mostepanenko, Ann. Phys. (N.Y.) 318,
37 (2005)

[11] F. Chen, U. Mohideen, G. L. Klimchitskaya, and V. M. Mostepanenko, Phys. Rev. A 72, 020101(R) (2005); 74, 022103 (2006).

[12] F. Chen, G. L. Klimchitskaya, V. M. Mostepanenko, and U. Mohideen, Phys. Rev. Lett. 97, 170402 (2006).

[13] F. Chen, G. L. Klimchitskaya, V. M. Mostepanenko, and U. Mohideen, Opt. Express 15, 4823 (2007); Phys. Rev. B 76, 035338 (2007).

[14] R. S. Decca, D. López, E. Fischbach, G. L. Klimchitskaya, D. E. Krause, and V. M. Mostepanenko, Phys. Rev. D 75, 077101 (2007); Eur. Phys. J. C 51, 963 (2007).

[15] G. Jourdan, A. Lambrecht, F. Comin, and J. Chevrier, EPL 85, 31001 (2009).

[16] H. B. Chan, Y. Bao, J. Zou, R. A. Cirelli, F. Klemens, W. M. Mansfield, and C. S. Pai, Phys. Rev. Lett. 101, 030401 (2008).

[17] J. Blocki, J. Randrup, W. J. Swiatecki, and C. F. Tsang, Ann. Phys. (N.Y.) 105, 427 (1977).

[18] C. C. Speake and C. Trenkel, Phys. Rev. Lett. 90, 160403 (2003).

[19] http://www.prhoffman.com/technical/scratch-dig.htm

[20] G. L. Klimchitskaya, U. Mohideen, and V. M. Mostepanenko, J. Phys. A: Math. Theor. 40, F339 (2007). 